\title{
Leveraging Image Analysis for High-Throughput Plant Phenotyping
}

\author{
Sruti Das Choudhury ${ }^{1,2 *}$, Ashok Samal ${ }^{2}$ and Tala Awada ${ }^{1,3}$ \\ ${ }^{1}$ School of Natural Resources, University of Nebraska-Lincoln, Lincoln, NE, United States, ${ }^{2}$ Department of Computer \\ Science and Engineering, University of Nebraska-Lincoln, Lincoln, NE, United States, ${ }^{3}$ Agricultural Research Division, \\ University of Nebraska-Lincoln, Lincoln, NE, United States
}

The complex interaction between a genotype and its environment controls the biophysical properties of a plant, manifested in observable traits, i.e., plant's phenome, which influences resources acquisition, performance, and yield. High-throughput automated image-based plant phenotyping refers to the sensing and quantifying plant traits non-destructively by analyzing images captured at regular intervals and with precision. While phenomic research has drawn significant attention in the last decade, extracting meaningful and reliable numerical phenotypes from plant images especially by considering its individual components, e.g., leaves, stem, fruit, and flower, remains a critical bottleneck to the translation of advances of phenotyping technology into genetic insights due to various challenges including lighting variations, plant rotations, and self-occlusions. The paper provides (1) a framework for plant phenotyping in a multimodal, multi-view, time-lapsed, high-throughput imaging system; (2) a taxonomy of phenotypes that may be derived by image analysis for better understanding of morphological structure and functional processes in plants; (3) a brief discussion on publicly available datasets to encourage algorithm development and uniform comparison with the state-of-the-art methods; (4) an overview of the state-of-the-art image-based high-throughput plant phenotyping methods; and (5) open problems for the advancement of this research field.

Keywords: high-throughput plant phenotyping, image analysis, multimodal image sequence, phenotype taxonomy, structural phenotype, physiological phenotype, temporal phenotype

*Correspondence:

Sruti Das Choudhury S.D.Choudhury@unl.edu

Specialty section:

This article was submitted to Technical Advances in Plant Science,

a section of the journal

Frontiers in Plant Science

Received: 13 November 2018 Accepted: 02 April 2019 Published: 24 April 2019

Citation:

Das Choudhury S, Samal A and Awada T (2019) Leveraging Image Analysis for High-Throughput Plant Phenotyping. Front. Plant Sci. 10:508.

doi: 10.3389/fpls.2019.00508

\section{INTRODUCTION}

The temporal variation in the plant's morphological and functional traits regulated by genotype and the environment plays a crucial role for the development of crops that impact both yield and quality (Lobos et al., 2017). High-throughput image-based plant phenotyping facilitates the computation of phenotypes by analyzing a large number of plants in short time interval with precision, nullifying the need for time-consuming physical human labor (Das Choudhury et al., 2018). The process is generally non-destructive, allowing the same traits to be quantified repeatedly at multiple times and scales during a plant's life cycle. It is an interdisciplinary research field involving computer science, biology, remote sensing, statistics, and genomics in the effort to link intricate plant phenotypes to genetic expression in order to meet current and emerging issues in agriculture relating to future food security under dwindling natural resources and projected climate variability and change.

Plants are not static but living organisms with constantly increasing complexity in shape, architecture, and appearance. Many plants alter leaf positioning (i.e., phyllotaxy) in response to 
light signals perceived through the photochrome in order to optimize light interception (Maddonni et al., 2002). In addition to variation in phyllotaxy, growth of individual leaves leads to self-occlusions and leaf crossovers over time posing challenges to image-based plant phenotyping analysis. Other challenges include variation in illumination, cluttered background, and change in zoom levels in the cameras often used to adjust for plant growth.

The state-of-the-art computer vision based approaches mainly focus on the structural and morphological aspects of a plant for computing $2 \mathrm{D}$ and $3 \mathrm{D}$ phenotypes. However, quantification of physiological processes in plant components, i.e., leaves, stem segments, flowers, fruits, etc., may show differential behavior as a function of environmental stress, and result in different leaf-level photosynthetic activity or carbohydrate content in a plant segment. Similarly, dynamic event-based phenotypes, i.e., identifying important events in plant life cycle, e.g., emergence timing of a new leaf, automated germination detection, timing of emergence detection of fruits and flowers, may provide important insights into the acclimation and adaptation strategies of plants. Thus, we present a novel taxonomy, beyond the well-studied class of structural phenotypes, to illustrate the vast potential of image analysis based methods to assist in understanding of insightful physiological and temporal phenotypes. To achieve this, full range of available imaging modalities and mechanisms must be used. Therefore, we propose a general computational framework for image-based plant phenotyping. We also summarize state-of-the-art image-based plant phenotyping methods with discussion on potential future developments in this field. To systematically evaluate and uniformly compare the methods, benchmark datasets are indispensable. Thus, the paper also provides a comprehensive summary of the publicly available datasets.

\section{HIGH-THROUGHPUT PLANT PHENOTYPING PLATFORM}

Figure 1 shows a schematic diagram for image-based highthroughput plant phenotyping platform. It analyses temporal image sequences of a group of plants (belonging to different genotypes) captured by multimodal cameras, i.e., visible light, fluorescent, near infrared, infrared and hyperspectral, from different viewing angles. The plants are imaged at regular intervals under various environmental conditions, e.g., drought, salinity, and thermal (Das Choudhury et al., 2016). Motivated by the high-throughput plant phenotyping platform presented in Fahlgren et al. (2015), we design a more advanced representation to explore computer vision based plant phenotyping algorithms in multiple dimensions, i.e., multimodal, multiview and temporal, regulated by genotypes under various environmental conditions. The image sequences captured by visible light camera are often used to compute structural or morphological phenotypes. Fluorescent, near infrared and infrared images are respectively used to analyze the chlorophyll, water, and temperature content of the plants. Thermal infrared imaging is used as proxy for a plant's temperature to detect differences in stomatal conductance as a measure of the plant response to the water status and transpiration rate for abiotic stress adaptation (Lei et al., 2014b). Hyperspectral imaging is uniquely suited to provide insights into the functional properties of plants, e.g., leaf tissue structure, leaf pigments, and water content (Mahlein et al., 2011) and stress resistance mechanisms (Wahabzada et al., 2016). The high-throughput plant phenotyping platforms presented by Rahaman et al. (2015) and Araus and Cairns (2014) provide emphasis on bridging gap between phenotype-genotype relationship from the molecular point of view.

\section{A TAXONOMY FOR PLANT PHENOTYPES}

We present a plant phenotypic taxonomy (Figure 2) which reflects plant phenotypes introduced in recent literature as well as the new challenges that require future research attention. The figure shows that the aboveground plant phenotypes are broadly classified into three categories, namely, structural, physiological, and temporal. The structural phenotypes refer to the morphological attributes of the plants, whereas the physiological phenotypes are related to traits that affect plant processes regulating growth and metabolism. Structural and physiological phenotypes are further divided into two groups: holistic and component. The holistic phenotypes consider the whole plant as a single object and compute its basic geometrical properties, e.g., height of the bounding rectangle to account for plant height, area of the convex-hull to account for plant size (Das Choudhury et al., 2017, 2018). Component phenotypes are computed by considering individual components of the plants, i.e., leaves, stem, flower, and fruit. Examples of component phenotypes include leaf length, chlorophyll content of each leaf, stem angle, flower size, and fruit volume (Gage et al., 2017; He et al., 2017; Das Choudhury et al., 2018; Yin et al., 2018; Zhou et al., 2019).

The different parts of plants grow non-uniformly over space and time. This non-uniformity in growth is also regulated by the genotypes. The temporal phenotypes are computed by analyzing a sequence of images. We propose two different types of temporal phenotypes: trajectory-based and eventbased. The structural and physiological phenotypes can be computed from a single image or a sequence of images to take into account of temporal phenotypic characteristics for better genetic variability, e.g., plant growth rate, leaf elongation rate, and trajectories of stem angle (Das Choudhury et al., 2016, 2018; Sun et al., 2018). It includes change in phenotypic traits as a function of time, time-rate of change in stem width and relative growth rates of different leaves. Eventbased phenotypes are based on specific events in a plant's life cycle, e.g., germination, emergence of a new leaf, flowering, etc. The timing of the events with reference to an origin (say planting date) is important in understanding a plant's behavior (Agarwal, 2017). While the primary phenotypes refer to one specific characteristic of a plant, derived phenotypes 
are composed of two or more primary phenotypes, i.e., biangular convex-hull area ratio to account for phyllotaxy and plant aspect ratio to provide information on canopy architecture (Das Choudhury et al., 2016).

\section{PUBLIC DATASETS}

Development and public dissemination of datasets are vital for research in image based plant phenotyping as it provides the broader computer vision research community access to datasets that they typically do not have the ability to generate. Also, standard datasets provide a common basis to compare the performance of plant phenotyping algorithms. We briefly summarize the datasets below that are publicly disseminated.

- Leaf segmentation challenge (LSC) dataset: LSC dataset is released to advance the state-of-the-art in leaf segmentation, counting, and tracking of rosette plants. The dataset consists of images of two plant species, i.e., Arabidopsis (Arabidopsis thaliana) and tobacco (Nicotiana tabacum) organized into three subsets. Subsets A1 (Ara2012) and A2 (Ara2013) consist of top-view time-lapse images of Arabidopsis thaliana rosettes. The total number of images in Ara2012 and Ara2013 are 150 and 5048, respectively. Subset A3 (Tobacco) consists of top-view stereo image sequences of tobacco (Nicotiana tabacum) plants captured hourly for 30 days. The LSC dataset is publicly available from http://www.plant-phenotyping.org/ CVPPP2014-challenge.

- Michigan State University Plant imagery dataset (MSUPID): The MSU-PID dataset (Cruz et al., 2016) consists of images of Arabidopsis (total $2160 \times 4$ images) and bean (total $325 \times 4$ images) captured with four types of calibrated cameras, i.e., fluorescent, infrared, RGB color and depth sensor to facilitate research in leaf segmentation, leaf counting, leaf alignment, leaf tracking, and 3D leaf reconstruction. A subset (576 $\times 4$ Arabidopsis images and $175 \times 2$ bean images) is annotated to provide ground-truth for leaf tip location, leaf segmentation, and leaf alignment. MSU-PID dataset is publicly available from http://cvlab.cse.msu.edu/multimodality-imagery-database-msu-pid.html Cruz et al. (2016).

- Panicoid Phenomap-1: To stimulate the development and evaluation of holistic phenotypes of panicoid grain crops, a public dataset called Panicoid Phenomap-1 is introduced in Das Choudhury et al. (2016). It consists of visible light image sequences of 40 genotypes including at least one representative accession from five panicoid grain crops: maize, sorghum, pearl millet, proso millet, and foxtail millet. The images are captured by the Lemnatec scanalyzer high-throughput plant phenotyping facility at the University of Nebraska-Lincoln (UNL), USA.

- University of Nebraska-Lincoln Component Plant Phenotyping Dataset (UNL-CPPD): A benchmark dataset called UNL-CPPD in introduced in Das Choudhury et al. (2018), to spur research in leaf detection and tracking, leaf segmentation, evaluation of holistic, and component phenotypes for maize and cereal crops with similar architecture, e.g., sorghum. UNL-CPPD includes human-annotated ground-truth along with the original image sequences to facilitate image-based component phenotyping analysis.

- Komatsuna Dataset: Komatsuna Dataset (Uchiyama et al., 2017) contains images of early growth stages of Komatsuna plants with a leaf annotation tool to facilitate 3D plant phenotyping analysis such as leaf segmentation, tracking, and reconstruction. A set of 5 Komatsuna plants are imaged every $4 \mathrm{~h}$ for 10 days using a RGB camera (Multiview dataset) and a RGB camera fitted with structured light depth camera (RGB$\mathrm{D}$ dataset). The dataset is freely available from http://limu.ait. kyushu-u.ac.jp/ agri/komatsuna/.

- University of Nebraska-Lincoln 3D Plant Phenotyping Dataset (UNL-3DPPD): This dataset consists of images of 20 maize and 20 sorghum plants for 10 side views to facilitate 3D plant phenotyping research. Plants were imaged once per day using the visible light camera of the UNL Lemnatec Scanalyzer 3D high-throughput phenotyping facility. Panicoid Phenomap-1, UNL-CPPD, and UNL-3DPPD can be freely downloaded from http://plantvision.unl.edu/.

- Deep Phenotyping Dataset: This dataset consists of 22 successive top-view image sequences of four accessions of Arabidopsis, i.e., Sf-2, Cvi, Landsberg, and Columbia, captured once daily to study temporal phenotypes for accession classification using convolutional neural network $(\mathrm{CNN})$, recurrent neural network and long-short term memory (LSTM) (Taghavi Namin et al., 2018). The dataset is augmented by rotating each image by $90^{\circ}$, $180^{\circ}$, and $270^{\circ}$ to avoid overfitting while training CNN. It can be freely downloaded from https://figshare.com/s/ e18a978267675059578f.

\section{RECENT ADVANCEMENTS IN IMAGE-BASED PLANT PHENOTYPING}

This section provides a brief description of the state-of-the-art methods to compute phenotypes from images organized using our taxonomy, i.e., structural, physiological, and temporal.

\subsection{Structural Phenotypes 5.1.1. 2D Phenotypes}

The method in Dellen et al. (2015) uses a leaf-shape model to detect each leaf of a tobacco plant. A graph-based tracking algorithm is used to track the detected leaves in a sequence to measure growth rate as a trajectory-based structural phenotype. Leaf alignment and tracking are formulated as two optimization problems in Yin et al. (2018) based on Chamfer matching and leaf template transformation from fluorescent videos for application in leaf-level photosynthetic capability estimation. Das Choudhury et al. (2018) introduced a set of new holistic and component phenotypes computed from 2D side view image sequences of maize plants, and demonstrated the temporal variations of these phenotypes regulated by genotypes. The method accepts plant image sequence as the input and produces a leaf status report containing the phenotypic information, i.e., the emergence timing, total number of leaves present at any point 



FIGURE 2 | High-throughput plant phenotyping platform. 
of time, total number of leaves emerged, the day on which a particular leaf stopped growing or lost, and the length and relative growth rate of individual leaves. The method in Das Choudhury et al. (2017) introduces an algorithm to compute stem angle, a potential measure for plants' susceptibility to lodging, based on graph-based plant architecture determination. A time series clustering analysis is used to summarize the temporal patterns of the stem angles into different groups to provide further insight into genotype specific behavior of the plants. Unlike the methods by Das Choudhury et al. $(2016,2018)$ which focus on vegetative stage phenotyping analysis of maize, the method by Brichet et al. (2017) develops a robot-assisted imaging pipeline to track the growths of ear and silks based on an ear detection algorithm. The genotypic variation in silk growth rate under drought stress is experimentally demonstrated.

It has been suggested that future progress in image-based plant phenotyping will require a combined effort in the domains of image processing for feature extraction and machine learning for data analysis (Tsaftaris et al., 2016). In recent times, machine learning techniques have gained popularity in high-throughput 2D phenotyping, e.g., in detection of branch shaking locations for robotic cherry harvesting (Amatya et al., 2017) and plant growth analysis (Navarro et al., 2016). Deep learning is an emerging field that promises unparalleled results on many data analysis problems. Building on artificial neural networks, deep approaches have many more hidden layers in the network, and hence have greater discriminative and predictive power. A deconvolutional network is used for segmenting the rosette leaves from the background, and then a CNN is used for leaf counting in the method by Aich and Stavness (2017). The method by Atanbori et al. (2018) re-architects four existing deep neural networks to create "Lite" CNN models in an attempt to reduce their parameters while avoiding overfitting for cost-effective solutions in plant phenotyping. The method also introduces a global hyper-parameter for efficient trade-off between parameter size and accuracy of "Lite" CNN models. The method by Pound et al. (2017) uses CNN to identify quantitative trait loci by classifying biologically relevant features such as root tips, leaf and ear tips, and leaf bases to determine root and shoot architecture.

An open source software tool based on CNN called Deep Plant Phenomics is proposed in Ubbens and Stavness (2017) to compute complex phenotypes from plant image sequences. ResNet50, a deep residual neural network, is used in Dobrescu et al. (2017) as a leaf prediction model to count the number of leaves of the rosette plants. In this method, leaf counting is modeled as a direct regression problem. A comprehensive summary of deep learning algorithms for identification, classification, quantification, and prediction of plant stress phenotypes is presented by Singh et al. (2018). Deep learning has applications in a variety of plant phenotyping tasks, e.g., plant stalk count and stalk width (Baweja et al., 2017), leaf counting in rosette plants (Giuffrida et al., 2018; Ubbens et al., 2018), maize tassel counting (Lu et al., 2017), cotton bloom detection (Xu et al., 2018), wheat spikes detection (Hasan et al., 2018), and rice panicle segmentation (Xiong et al., 2017).

\subsubsection{D Phenotypes}

The plants exhibit increasing architectural complexity with time due to self-occlusions and leaf crossovers, which pose challenges to accurate estimation of 2D component phenotypes, e.g., leaf length, stem angle, and leaf curvature. To overcome these challenges, attempts have been made to reconstruct a 3D model of a plant for accurate estimation of phenotypes. A shape-from-silhouette method is used in Golbach et al. (2016) to reconstruct the 3D model of a tomato seedling from multi-view images. An algorithm for depth imagingbased detection of muskmelon plant for phenotyping in the greenhouse is proposed in Lei et al. (2014a). A detailed review on 3D reconstruction techniques for shoot topology for applications in phenotyping is provided in Gibbs et al. (2016). The method in Liu et al. (2017) uses the structure from motion method to reconstruct the 3D model of a plant using images from multiple side views. The method also deducted the optimal number of images needed for reconstructing a high-quality model. Most cereal crops, e.g., rice, wheat, and maize, have thin ribbon-like architectures with lack of textural surfaces, which limits the success of $3 \mathrm{D}$ reconstruction to early growth stages where the distance between the camera and the plant is relatively small facilitating accurate camera calibration.

$3 \mathrm{D}$ reconstruction of soybean canopies using multisource imaging is presented in Guan et al. (2018). A densitybased spatial clustering of applications with noise removal is used to extract canopy information from the raw 3D point cloud. The method in Scharr et al. (2017) uses a voxelbased volume carving for $3 \mathrm{D}$ reconstruction of maize shoot to compute leaf-level traits. Depth cameras capture 3D information from plants to segment leaves and reconstruct plant models (Chéné et al., 2012). A semi-automated software pipeline is developed to reconstruct a 3D plant model from the images captured by a depth camera in McCormick et al. (2016). Standard shoot phenotypes such as shoot height, leaf angle, leaf length, and shoot compactness are measured from 3D plant reconstructions to characterize the shoot architecture. The Microsoft Kinect, originally designed for computer gaming environments, has found applications in 3D plant phenotyping in recent times (Chéné et al., 2012; Paulus et al., 2014; Polder and Hofstee, 2014.)

The method in Polder and Hofstee (2014) fuses RGB and depth image captured by Microsoft Kinect sensor to segment muskmelon plants in the greenhouse with cluttered background. The method in Srivastava et al. (2017) provides an algorithm for 3D model reconstruction of wheat plants with occluded leaves, and uses deep learning for drought stress characterization. The remote sensing technology using light detection and ranging (LiDAR) is hypothesized to dominate the future generation of plant phenotyping analysis in outdoor environments Lin (2015). The methods in Sun et al. (2017, 2018) compute morphological traits of the cotton plants, e.g., canopy height, projected canopy area, and plant volume, based on 3D model reconstruction of the plants using top view images captured by LiDAR and real-time kinematic global positioning system (RTK-GPS). 


\subsection{Physiological Phenotypes}

A comprehensive review on close proximal assessment of functional dynamics of plants using hyperspectral image analysis is provided in Mishra et al. (2017). Water scarcity causes serious crop losses in agriculture. Global climate change and growing population require research advancement in the understanding of plant resistance mechanisms to drought stress for improved crop yield with minimum resource utilization. The method in Römer et al. (2012) uses a simplex volume maximization technique for early drought stress detection using hyperspectral image sequences of barley plants. Recent developments in hyperspectral imaging for assessment of food quality and safety are discussed in Huang et al. (2014), Lu et al. (2017), and Rungpichayapichet et al. (2017). Hyperspectral image analysis has been used to investigate physiological processes, e.g., determination of salt stress in wheat based on vector-wise similarity measurement (Moghimi et al., 2018), early detection of abiotic stresses (Mohd et al., 2018), disease detection to prevent yield losses (Wahabzada et al., 2016), and early yield prediction (González-Sanchez et al., 2014).

A Matlab based software called SK-UTALCA is introduced in Lobos and Poblete-Echeverría (2017) for applications in plant breeding, precision agriculture, crop protection, ecophysiology plant nutrition, and soil fertility by analyzing high-resolution spectral reflectances. A comprehensive overview of the machine learning techniques in the identification, classification, quantification, and prediction of various biotic and abiotic stress phenotypes are provided in Singh et al. (2016). The method by Raza et al. (2014) combines information extracted from thermal and visible light images, and uses support vector machine (SVM) and Gaussian processes (GP) to identify regions of spinach canopy showing a response to soil water deficit. A review of machine learning approaches including back-propagation neural networks, GP, SVM, rotation forest, CNN and LSTM, for crop yield prediction and nitrogen status estimation in precision agriculture is presented in Chlingaryan et al. (2018).

\subsection{Temporal Phenotypes}

The method in Das Choudhury et al. (2018) uses line graphs to represent the trajectories of component phenotypes, i.e., leaf length, integral leaf-skeleton area, mid-leaf curvature, apex curvature and stem angle, as a function of time in order to demonstrate the genetic influence on the temporal variations of these phenotypes. A novel method for plant emergence detection using adaptive hierarchical segmentation and optical flow based tracking is introduced in Agarwal (2017). The efficacy of the method is demonstrated based on experimental analysis on a dataset consisting of images captured at every $2-5 \mathrm{~min}$ intervals starting immediately after planting the seeds.

\section{OPEN PROBLEMS}

Most of the research in image-based plant phenotyping is focused on structural and mainly holistic physiological phenotypes.
However, timing determination of important events in the life of a plant, e.g., germination, emergence and senescence of leaves, emergence of flowers and fruits, can provide crucial information about plant's growth and response to biotic and abiotic stresses. Timing detection of such events using computer vision techniques remain an important open problem.

Most of the phenotypes have focused on either aboveground or belowground phenotypes. We propose a new category of phenotype, called integrated phenotype, that establishes relationship between above- and belowground phenotypes affected by abiotic and biotic stresses. Primary root growth is inhibited during P-limitation, and its length determines plant's capability to access stored water in the deeper layers of the soil substratum (Prasad et al., 2012; Paez-Garcia et al., 2015). A new integrated phenotype, e.g., the ratio of stem height to primary root length as a function of time, may be investigated for increased yield of stress tolerant crops by enhancing the capacity of the plant for soil exploration and, thus, water and nutrient acquisition. Algorithms to compute them from plant imagery need to be developed.

Efforts have been made in early detection of stress, e.g., drought and mineral (Kim et al., 2011; Chen and Wang, 2014; van Maarschalkerweerd and Husted, 2015). However, future work is required in the investigation of phenotypes that characterize the prorogation of stress as a function time and also categorize stress into different stages, e.g., slight, moderate, extreme, and exceptional. The speed of recovery from the stresses regulated by genotypes is also an open challenge. The method by Uga et al. (2013) demonstrates that controlling root growth angle contributes to drought tolerance. Hence, we propose a new integrated phenotype to quantify speed of drought recovery from different stress levels in relation to controlled root angle.

Extending the manual leaf tracking method in Das Choudhury et al. (2018), we formulate a new problem called visual growth tracking (i.e., tracking of different parts of an object that grows at different rates over time) using plant image sequences for automated growth monitoring of different components of plants, i.e., leaves, flowers, and fruits.

Computing phenotypes from 2D images (e.g., stem angle) are inherently error-prone as they are dependent on accurate camera views. They are deficient too because the plant is a $3 \mathrm{D}$ structure and any projection onto $2 \mathrm{D}$ plane results in loss of information. Thus, new and innovative 3D phenotypes, both holistic and component, based on accurate reconstruction of the model of a plant must be developed to accurately characterize its properties. 3D plant model reconstruction has been successful for early growth stages with less architectural complexity (Golbach et al., 2016). Advanced 3D plant model reconstruction algorithms are yet to be developed for entire life cycle of plants covering vegetative and reproductive stages for computation of derived phenotypes, e.g., stem cross-section area as a function of plant height at its different stages, 3D leaf area to leaf length ratio, 3D leaf curvature to leaf length ratio, carbohydrate content of stem with respect to stem volume and plant temperature to convex-hull volume ratio at different stages of stressed plants. 


\section{AUTHOR CONTRIBUTIONS}

SD contributed as the first author in reviewing the literature, compiling the information, preparing the review, and writing the manuscript. AS and TA contributed as co-authors to outline the sections. They critically reviewed the manuscript and provided constructive feedback throughout the process.

\section{REFERENCES}

Agarwal, B. (2017). Detection of Plant Emergence Based on Spatio Temporal Image Sequence Analysis. Masters Thesis, University of Nebraska-Lincoln.

Aich, S., and Stavness, I. (2017). "Leaf counting with deep convolutional and deconvolutional networks," in The IEEE International Conference on Computer Vision (ICCV) Workshops (Venice), 2080-2089.

Amatya, S., Karkee, M., Zhang, Q., and Whiting, M. D. (2017). Automated detection of branch shaking locations for robotic cherry harvesting using machine vision. Robotics 6:31. doi: 10.3390/robotics 6040031

Araus, J. L., and Cairns, J. E. (2014). Field high-throughput phenotyping: the new crop breeding frontier. Trends Plant Sci. 19, 52-61. doi: 10.1016/j.tplants.2013.09.008

Atanbori, J., Chen, F., French, A. P., and Pridmore, T. P. (2018). "Towards lowcost image-based plant phenotyping using reduced-parameter CNN," in BMVC Workshop on Computer Vision Problmes in Plant Phenotyping (Newcastle).

Baweja, H. S., Parhar, T., Mirbod, O., and Nuske, S. (2017). "Stalknet: a deep learning pipeline for high-throughput measurement of plant stalk count and stalk width," in Proceedings in Advanced Robotics (Zurich: Springer), 271-284.

Brichet, N., Fournier, C., Turc, O., Strauss, O., Artzet, S., Pradal, C., et al. (2017). A robot-assisted imaging pipeline for tracking the growths of maize ear and silks in a high-throughput phenotyping platform. Plant Methods 13:96. doi: 10.1186/s13007-017-0246-7

Chen, L., and Wang, K. (2014). Diagnosing of rice nitrogen stress based on static scanning technology and image information extraction. J. Soil Sci. Plant Nutr. 14, 382-393. doi: 10.4067/S0718-95162014005000030

Chéné, Y., Rousseau, D., Lucidarme, P., Bertheloot, J., Caffier, V., Morel, P., et al. (2012). On the use of depth camera for 3D phenotyping of entire plants. Comput. Electron. Agric. 82, 122-127. doi: 10.1016/j.compag.2011. 12.007

Chlingaryan, A., Sukkarieh, S., and Whelan, B. (2018). Machine learning approaches for crop yield prediction and nitrogen status estimation in precision agriculture: a review. Comput. Electron. Agric. 151, 61-69. doi: 10.1016/j.compag.2018.05.012

Cruz, J. A., Yin, X., Liu, X., Imran, S. M., Morris, D. D., Kramer, D. M., et al. (2016). Multi-modality imagery database for plant phenotyping. Mach. Vis. Appl. 27, 735-749. doi: 10.1007/s00138-015-0734-6

Das Choudhury, S., Bashyam, S., Qiu, Y., Samal, A., and Awada, T. (2018). Holistic and component plant phenotyping using temporal image sequence. Plant Methods 14:35. doi: 10.1186/s13007-018-0303-x

Das Choudhury, S., Goswami, S., Bashyam, S., Samal, A., and Awada, T. (2017). "Automated stem angle determination for temporal plant phenotyping analysis," in ICCV Workshop on Computer Vision Problmes in Plant Phenotyping (Venice: ACM Press), 41-50.

Das Choudhury, S., Stoerger, V., Samal, A., Schnable, J. C., Liang, Z., and Yu, J.-G. (2016). "Automated vegetative stage phenotyping analysis of maize plants using visible light images," in KDD Workshop on Data Science for Food, Energy and Water (San Francisco, CA).

Dellen, B., Scharr, H., and Torras, C. (2015). Growth signatures of rosette plants from time-lapse video. IEEE ACM Trans. Comput. Biol. Bioinformatics 12, 1470-1478. doi: 10.1109/TCBB.2015.2404810

Dobrescu, A., Valerio Giuffrida, M., and Tsaftaris, S. A. (2017). "Leveraging multiple datasets for deep leaf counting," in The IEEE International Conference on Computer Vision (ICCV) Workshops (Venice), 2072-2079.

\section{FUNDING}

This research was supported by the Nebraska Agricultural Experiment Station with funding from the Hatch Act capacity funding program (Accession Number 1011130) from the USDA National Institute of Food and Agriculture. This study was also supported by the McIntire Stennis funds, USDA (NEB-38-114).

Fahlgren, N., Gehan, M. A., and Baxter, I. (2015). Lights, camera, action: high throughput plant phenotyping is ready for a close-up. Curr. Opin. Plant Biol. 24, 93-99. doi: 10.1016/j.pbi.2015.02.006

Gage, J. L., Miller, N. D., Spalding, E. P., Kaeppler, S. M., and de Leon, N. (2017). Tips: a system for automated image-based phenotyping of maize tassels. Plant Methods 13:21. doi: 10.1186/s13007-017-0172-8

Gibbs, J., Pound, M., French, A., Wells, D., Murchie, H. E., and Pridmore, T. (2016). Approaches to three-dimensional reconstruction of plant shoot topology and geometry. Funct. Plant Biol. 44, 62-75. doi: 10.1071/FP16167

Giuffrida, M. V., Doerner, P., and Tsaftaris, S. A. (2018). Pheno-deep counter: a unified and versatile deep learning architecture for leaf counting. Plant J.96, 880-890. doi: 10.1111/tpj.14064

Golbach, F., Kootstra, G., Damjanovic, S., Otten, G., and van de Zedde, R. (2016). Validation of plant part measurements using a 3D reconstruction method suitable for high-throughput seedling phenotyping. Mach. Vis. Appl. 27, 663680. doi: 10.1007/s00138-015-0727-5

González-Sanchez, A., Frausto-Solis, J., and Ojeda, W. (2014). Predictive ability of machine learning methods for massive crop yield prediction. Spanish J. Agric. Res. 12, 313-328. doi: 10.5424/sjar/2014122-4439

Guan, H., Liu, M., Ma, X., and Yu, S. (2018). Three-dimensional reconstruction of soybean canopies using multisource imaging for phenotyping analysis. Remote Sens. 10:1206. doi: 10.3390/rs10081206

Hasan, M. M., Chopin, J. P., Laga, H., and Miklavcic, S. J. (2018). Detection and analysis of wheat spikes using convolutional neural networks. Plant Methods 14:100. doi: 10.1186/s13007-018-0366-8

He, J. Q., Harrison, R. J., and Li, B. (2017). A novel 3d imaging system for strawberry phenotyping. Plant Methods 13:93. doi: 10.1186/s13007-017-0243-x

Huang, H., Liu, L., and Ngadi, M. O. (2014). Recent developments in hyperspectral imaging for assessment of food quality and safety. Sensors 14, 7248-7276. doi: 10.3390/s140407248

Kim, Y., Glenn, D. M., Park, J., Ngugi, H. K., and Lehman, B. L. (2011). Hyperspectral image analysis for water stress detection of apple trees. Comput. Electron. Agric. 77, 155-160. doi: 10.1016/j.compag.2011.04.008

Lei, L., Qin, Z., and Danfeng, H. (2014a). "Depth imaging-based detection of muskmelon plant for phenotyping in the greenhouse," in $A S A B E$ and CSBE/SCGAB Annual International Meeting (Montreal, QC), 153-159.

Lei, L., Qin, Z., and Danfeng, H. (2014b). A review of imaging techniques for plant phenotyping. Sensors 14, 20078-20111. doi: 10.3390/s141120078

Lin, Y. (2015). Lidar: an important tool for next-generation phenotyping technology of high potential for plant phenomics? Comput. Electron. Agric. 119, 61-73. doi: 10.1016/j.compag.2015.10.011

Liu, S., Acosta-Gamboa, L. M., Huang, X., and Lorence, A. (2017). Novel low cost 3D surface model reconstruction system for plant phenotyping. J. Imaging 3:39. doi: 10.3390/jimaging3030039

Lobos, G. A., Camargo, A. V., del Pozo, A., Araus, J. L., Ortiz, R., and Doonan, J. H. (2017). Editorial: Plant phenotyping and phenomics for plant breeding. Front. Plant Sci. 8:2181. doi: 10.3389/fpls.2017.02181

Lobos, G. A., and Poblete-Echeverría, C. (2017). Spectral knowledge (sk-utalca): software for exploratory analysis of high-resolution spectral reflectance data on plant breeding. Front. Plant Sci. 7:1996. doi: 10.3389/fpls.2016.01996

Lu, Y., Huang, Y., and Lu, R. (2017). Innovative hyperspectral imaging-based techniques for quality evaluation of fruits and vegetables: a review. Appl. Sci. 7:189. doi: 10.3390/app7020189

Maddonni, G. A., Otegui, M. E., Andrieu, B., Chelle, M., and Casal, J. J. (2002). Maize leaves turn away from neighbors. Plant Physiol. 130, 1181-1189. doi: $10.1104 /$ pp.009738 
Mahlein, A.-K., Oerke, E.-C., Steiner, U., and Dehne, H.-W. (2011). Recent advances in sensing plant diseases for precision crop protection. Eur. J. Plant Pathol. 133, 197-209. doi: 10.1007/s10658-011-9878-z

McCormick, R. F., Truong, S. K., and Mullet, J. E. (2016). 3D sorghum reconstructions from depth images identify QTL regulating shoot architecture. Plant Physiol. 172, 823-834. doi: 10.1104/pp.16.00948

Mishra, P., Asaari, M. S. M., Herrero-Langreo, A., Lohumi, S., Diezma, B., and Scheunders, P. (2017). Close range hyperspectral imaging of plants: a review. Biosyst. Eng. 164, 49-67. doi: 10.1016/j.biosystemseng.2017.09.009

Moghimi, A., Yang, C., Miller, M. E., Kianian, S. F., and Marchetto, P. M. (2018). A novel approach to assess salt stress tolerance in wheat using hyperspectral imaging. Front. Plant Sci. 9:1182. doi: 10.3389/fpls.2018.01182

Mohd, S., mohd asaari mohd shahrimie, Puneet, M., Stien, M., Stijn, D., Dirk, I., Nathalie, W., and Paul, S. (2018). Close-range hyperspectral image analysis for the early detection of stress responses in individual plants in a high-throughput phenotyping platform. ISPRS J. Photogrammetr. Remote Sens. 138, 121-138. doi: 10.1016/j.isprsjprs.2018.02.003

Navarro, P. J., Pérez, F., Weiss, J., and Egea-Cortines, M. (2016). Machine learning and computer vision system for phenotype data acquisition and analysis in plants. Sensors 16:641. doi: 10.3390/s16050641

Paez-Garcia, A., Motes, C. M., Scheible, W.-R., Chen, R., Blancaflor, E. B., and Monteros, M. J. (2015). Root traits and phenotyping strategies for plant improvement. Plants 4, 334-355. doi: 10.3390/plants4020334

Paulus, S., Behmann, J., Mahlein, A.-K., Plümer, L., and Kuhlmann, H. (2014). Low-cost 3D systems: suitable tools for plant phenotyping. Sensors $14,3001-$ 3018. doi: 10.3390/s140203001

Polder, G., and Hofstee, J. (2014). "Phenotyping large tomato plants in the greenhouse using a 3D light-field camera," in ASABE and CSBE/SCGAB Annual International Meeting (Montreal, QC), 153-159.

Pound, M. P., Atkinson, J. A., Townsend, A. J., Wilson, M. H., Griffiths, M., Jackson, A. S., et al. (2017). Deep machine learning provides state-of-theart performance in image-based plant phenotyping. Gigascience 6, 1-10. doi: 10.1093/gigascience/gix083

Prasad, S. S., Wasson, A., Rebetzke, G., Kirkegaard, J., Richards, R., Watt, M., et al. (2012). Traits and selection strategies to improve root systems and water uptake in water-limited wheat crops. J. Exp. Bot. 63, 3485-3498. doi: $10.1093 /$ jxb/ers111

Rahaman, M., Chen, D., Gillani, Z., Klukas, C., and Chen, M. (2015). Advanced phenotyping and phenotype data analysis for the study of plant growth and development. Front. Plant Sci. 6:619. doi: 10.3389/fpls.2015.00619

Raza, S. E., Smith, H. K., Clarkson, G. J. J., Taylor, G., Thompson, A. J., Clarkson, J., et al. (2014). Automatic detection of regions in spinach canopies responding to soil moisture deficit using combined visible and thermal imagery. PLOS ONE 9:e97612. doi: 10.1371/journal.pone.0097612

Römer, C., Wahabzada, M., Ballvora, A., Pinto, F., Rossini, M., Panigada, C., et al. (2012). Early drought stress detection in cereals: simplex volume maximization for hyperspectral image analysis. Funct. Plant Biol. 39, 878-890. doi: 10.1071/FP12060

Rungpichayapichet, P., Nagle, M., Yuwanbun, P., Khuwijitjaru, P., Mahayothee, B., and Müller, J. (2017). Prediction mapping of physicochemical properties in mango by hyperspectral imaging. Biosyst. Eng. 159, 109-120. doi: 10.1016/j.biosystemseng.2017.04.006

Scharr, H., Briese, C., Embgenbroich, P., Fischbach, A., Fiorani, F., and Müller-Linow, M. (2017). Fast high resolution volume carving for 3D plant shoot reconstruction. Front. Plant Sci. 8:1680. doi: 10.3389/fpls.2017. 01680

Singh, A., Ganapathysubramanian, B., Singh, A. K., and Sarkar, S. (2016). Machine learning for high-throughput stress phenotyping in plants. Trends Plant Sci. 21, 110-124. doi: 10.1016/j.tplants.2015.10.015

Singh, A. K., Ganapathysubramanian, B., Sarkar, S., and Singh, A. (2018). Deep learning for plant stress phenotyping: trends and future perspectives. Trends Plant Sci. 23, 883-898. doi: 10.1016/j.tplants.2018.07.004
Srivastava, S., Bhugra, S., Lall, B., and Chaudhury, S. (2017). "Drought stress classification using 3D plant models," in 2017 IEEE International Conference on Computer Vision Workshops (ICCVW) (Venice), 2046-2054.

Sun, S., Li, C., and Paterson, A. H. (2017). In-field high-throughput phenotyping of cotton plant height using lidar. Remote Sens. 9:377. doi: 10.3390/rs90 40377

Sun, S., Li, C., Paterson, A. H., Jiang, Y., Xu, R., Robertson, J. S., et al. (2018). Infield high throughput phenotyping and cotton plant growth analysis using lidar. Front. Plant Sci. 9:16. doi: 10.3389/fpls.2018.00016

Taghavi Namin, S., Esmaeilzadeh, M., Najafi, M., Brown, T. B., and Borevitz, J. O. (2018). Deep phenotyping: deep learning for temporal phenotype/genotype classification. Plant Methods 14:66. doi: 10.1186/s13007-0180333-4

Tsaftaris, S. A., Minervini, M., and Scharr, H. (2016). Machine learning for plant phenotyping needs image processing. Mach. Vis. Appl. 21, 989-991. doi: 10.1016/j.tplants.2016.10.002

Ubbens, J., Cieslak, M., Prusinkiewicz, P., and Stavness, I. (2018). The use of plant models in deep learning: an application to leaf counting in rosette plants. Plant Methods 14:6. doi: 10.1186/s13007-018-0273-z

Ubbens, J. R. and Stavness, I. K. (2017). Deep plant phenomics: a deep learning platform for complex plant phenotyping tasks. Front. Plant Sci. 8:1190. doi: $10.3389 /$ fpls.2017.01190

Uchiyama, H., Sakurai, S., Mishima, M., Arita, D., Okayasu, T., Shimada, A., et al. (2017). "An easy-to-setup 3D phenotyping platform for komatsuna dataset," in The IEEE International Conference on Computer Vision (ICCV) Workshops (Venice), 2038-2045.

Uga, Y., Sugimoto, K., Ogawa, S., Rane, J., Ishitani, M., Hara, N., et al. (2013). Control of root system architecture by deeper rooting 1 increases rice yield under drought conditions. Nat. Genet. 45:9. doi: 10.1038/ng.2725

van Maarschalkerweerd, M., and Husted, S. (2015). Recent developments in fast spectroscopy for plant mineral analysis. Front. Plant Sci. 6:169. doi: 10.3389/fpls.2015.00169

Wahabzada, M., Mahlein, A.-K., Bauckhage, C., Steiner, U., Oerke, E.C., and Kersting, K. (2016). Plant phenotyping using probabilistic topic models: uncovering the hyperspectral language of plants. Sci. Rep. 6:22482. doi: $10.1038 /$ srep22482

Xiong, X., Duan, L., Liu, L., Tu, H., Yang, P., Wu, D., et al. (2017). Panicleseg: a robust image segmentation method for rice panicles in the field based on deep learning and superpixel optimization. Plant Methods 13:104. doi: 10.1186/s13007-017-0254-7

Xu, R., Li, C., Paterson, A. H., Jiang, Y., Sun, S., and Robertson, J. S. (2018). Aerial images and convolutional neural network for cotton bloom detection. Front. Plant Sci. 8:2235. doi: 10.3389/fpls.2017.02235

Yin, X., Liu, X., Chen, J., and Kramer, D. M. (2018). Joint multi-leaf segmentation, alignment, and tracking for fluorescence plant videos. IEEE Trans. Pattern Anal. Mach. Intell. 40, 1411-1423. doi: 10.1109/TPAMI.2017.2728065

Zhou, Y., Srinivasan, S., Mirnezami, S. V., Kusmec, A., Fu, Q., Attigala, L., et al. (2019). Semiautomated feature extraction from rgb images for sorghum panicle architecture gwas. Plant Physiol. 179, 24-37. doi: 10.1104/pp.18.00974

Conflict of Interest Statement: The authors declare that the research was conducted in the absence of any commercial or financial relationships that could be construed as a potential conflict of interest.

Copyright (C) 2019 Das Choudhury, Samal and Awada. This is an open-access article distributed under the terms of the Creative Commons Attribution License (CC BY). The use, distribution or reproduction in other forums is permitted, provided the original author(s) and the copyright owner(s) are credited and that the original publication in this journal is cited, in accordance with accepted academic practice. No use, distribution or reproduction is permitted which does not comply with these terms. 